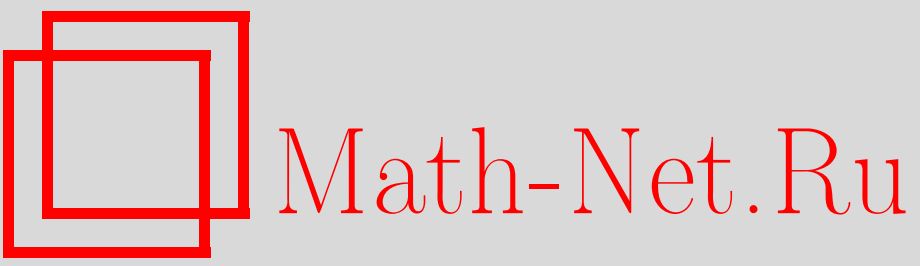

В. А. Андреев, В. И. Манько, Неравенство Белла для двучастичных смешанных спиновых состояний, ТМФ, 2004, том 140, номер 2, 284-296

DOI: https://doi.org/10.4213/tmf87

Использование Общероссийского математического портала Math-Net.Ru подразумевает, что вы прочитали и согласны с пользовательским соглашением

http://www . mathnet.ru/rus/agreement

Параметры загрузки:

IP : 54.205 .225 .156

26 апреля 2023 г., 11:28:00 
ТЕОРЕТИЧЕСКАЯ

И МАТЕМАТИЧЕСКАЯ

ФИЗИКА

Том 140, № 2

август, 2004

(C) 2004 г

В. А. Андреев* , В.И. Манько*

\section{НЕРАВЕНСТВО БЕЛЛА ДЛЯ ДВУЧАСТИЧНЫХ СМЕШАННЫХ СПИНОВЫХ СОСТОЯНИЙ}

В рамках традиционной квантовой механики и теории скрытых переменных построены неравенства Белла-Клаузера-Хорне-Шимони-Хольта (БКХШХ) для двухчастичных смешанных спиновых состояний. Рассматриваются два случая для векторов $\vec{a}$, $\vec{b}, \vec{c}, \vec{d}$, задающих направления, на которые проецируются спины частиц, образующих коррелированную пару. В первом случае эти векторы лежат в одной плоскости, во втором - ориентированы произвольно. Проведено сравнение полученных неравенств и показано, что для смешанных состояний разница в предсказаниях двух теорий меньше, чем для чистых состояний. Обнаружено, что для некоторых специальных состояний неравенства, полученные в рамках квантовой механики и теории скрытых переменных, совпадают; в частности, это верно для смешанных состояний, образованных чистыми факторизуемыми состояниями. Обсуждается сходство и различие между соотношениями неопределенностей и неравенствами Белла. Перечислены все состояния, для которых правая часть неравенства БКХШХ тождественно равна нулю.

Ключевые слова: квантовая механика, скрытые переменные, неравенство Белла, смешанные состояния, спиновые состояния, квантовая томография.

\section{1. ВВЕДЕНИЕ}

Отличительной чертой квантовой механики (KM) является то, что в ней явным образом присутствует неопределенность измеряемых величин. Наличие такой неопределенности приводит к тому, что некоторые закономерности в КМ формулируются в виде неравенства. Самым известным из этих неравенств является соотношение неопределенностей Гайзенберга [1]:

$$
\Delta x \Delta p \geqslant \frac{\hbar}{2}
$$

Оно связывает между собой "неопределенности" измерений координаты и импульса. Аналогичные неравенства сушествуют и для других пар канонически сопряженных переменных, а также для более широкого класса наблюдаемых, которым соответствуют некоммутирующие операторы. Неравенства такого типа отражают тот факт, что значения некоторых наблюдаемых не могут быть измерены одновременно с произвольной точностью.

* Физический институт им. П. Н. Лебедева РАН, Москва, Россия. E-mail: andr@sci.lebedev.ru; manko@sci.lebedev.ru 
Другой тип неравенств в КМ был обнаружен Беллом [2]. Они отражают тот факт, что большинство предсказаний в КМ носит вероятностный характер. Как правило, мы не можем предсказать точный результат измерения, а можем только вычислить вероятности, с которыми результат измерения принимает те или иные значения.

Вероятностная природа этих неравенств широко обсуждается в современной литературе [3]. При этом важным моментом является их формулировка в терминах случайных функций. Такой подход позволяет выявить принципиальные различия между вероятностными структурами традиционной КМ и альтернативными моделями, использующими скрытые переменные (СП). На наш взгляд, именно вероятностная трактовка данной проблематики придает ей актуальность. Действительно, если исходить только из задачи описания квантовых явлений, то обычная КМ вполне с этим справляется, а модель СП противоречит эксперименту. Но если посмотреть на эту модель как на специальную математическую вероятностную теорию со своей структурой, методами и возможными областями применения, то оказывается естественным рассмотреть ее вместе и наравне с КМ в рамках единой теоретико-вероятностной конструкции, и в рамках данной конструкции изучать и сравнивать их предсказания [4].

В разделе 2 мы проследим сходство структуры соотношений неопределенностей и обобщенных неравенств Белла для смешанных состояний. В разделе 3 мы в рамках КМ и модели СП построим и сравним между собой неравенства Белла-Клаузера-ХорнеШимони-Хольта (БКХШХ) [5] для смешанных двучастичных состояний. В разделе $4 \mathrm{c}$ помошью прямого вычисления покажем, что для разделяемых состояний эти неравенства совпадают друг с другом.

\section{2. СООТНОШЕНИЯ НЕОПРЕДЕЛЕННОСТЕЙ ДЛЯ СМЕШАННЫХ СОСТОЯНИЙ}

Соотношения неопределенностей и неравества Белла имеют, вообше говоря, разную природу. Однако в самое последнее время появились работы, в которых делаются попытки установить связь между ними. Как известно, при анализе неравенств Белла основное внимание уделяется запутанным состояниям, на которых и проявляются различия в предсказаниях между традиционной КМ и моделью СП. В работе [6] двучастичные спиновые состояния анализируются с точки зрения соотношений неопределенностей. Показано, что для факторизуемых и разделяемых двучастичных состояний неопределенность некоторых наблюдаемых равна сумме неопределенностей этих наблюдаемых для одночастичных состояний. Но для запутанных состояний неопределенность таких наблюдаемых может оказаться меньше суммы неопределеннностей для одночастичных состояний. Этот факт называют "нарушением соотношений неопределенностей”. При этом минимальная неопределенность отвечает чистым запутанным состояниям. Смешанным запутанным состояниям соответствуют промежуточные значения неопределенностей, величины которых зависят от структуры состояния. Схожая картина наблюдается и для неравенств Белла: их максимальное нарушение происходит на чистых запутанных состояниях, а с помошью смешанных запутанных состояний можно получить все другие неравенства с меньшими правыми частями. Характерно и то, что в работе [6] в качестве примера "нарушения соотношений неопределенностей" рассмат- 
ривается состояние Вернера [7], которое шшроко обсуждается и при анализе нарушений неравенств Белла.

Приведем еше один пример, указывающий на сходство между соотношениями неопределенностей и неравенствами Белла. Он также реализуется на смешанных состояниях. Пусть мы имеем дело со смешанным состоянием и нам известно, какие чистые состояния и с какими вероятностями в него входят. Тогда известно, как модифицируются соотношения неопределенностей при переходе от чистых состояний к смешанным. Действительно, рассмотрим соотношение неопределенностей для координаты и импульса. Кеннардом [8] и Вейлем [9] было показано, что для произвольных чистых состояний такое соотношение неопределенностей имеет вид

$$
\sigma_{x} \sigma_{p} \geqslant \frac{1}{4} \hbar^{2}
$$

Здесь $\sigma_{x}, \sigma_{p}$ - дисперсии соответственно координаты и импульса. В работе [9] было установлено, что для смешанных состояний, описываемых матрицей плотности $\rho$, вместо соотношения (2) выполняется соотношение

$$
\sigma_{x} \sigma_{p} \geqslant \frac{1}{4} \hbar^{2} \Phi(\mu)^{2} .
$$

Здесь $\mu=\operatorname{Sp} \rho^{2}$ - величина, называемая "пьюрити". Для чистых состояний $\mu=1$, а для смешанных $\mu \leqslant 1$. Функция $\Phi(\mu)(\Phi(1)=1)$ монотонно растет при уменьшении параметра $\mu$. Ее явный вид был найден в работе [10]. Таким образом, соотношение неопределенностей для смешанных состояний получается из соотношения неопределенностей для чистых состояний путем умножения правой части на функцию от величины $\mu$.

Аналогичную ситуацию можно наблюдать и в случае неравенств Белла. Поясним это утверждение на примере. Известно, что для чистых запутанных состояний выполняется КМ-неравенство БКХШХ [5]

$$
|E(\vec{a}, \vec{b})+E(\vec{a}, \vec{c})+E(\vec{d}, \vec{b})-E(\vec{d}, \vec{c})| \leqslant 2 \sqrt{2},
$$

где $E(\vec{a}, \vec{b})$ - среднее значение спиновой корреляции (см. ниже формулы $(20),(21))$.

Рассмотрим смешанное состояние, образованное двумя чистыми состояниями $\Psi_{1}, \Psi_{2}$, которые входят в него с вероятностями $w_{1}, w_{2}$. Волновые функции чистых состояний имеют вид

$$
\begin{gathered}
\Psi_{1}=\Psi_{++}, \quad \Psi_{2}=\gamma \Psi_{+-}+\delta \Psi_{-+} \\
|\gamma|^{2}+|\delta|^{2}=1, \quad w_{1}+w_{2}=1
\end{gathered}
$$

Матрица плотности такого состояния

$$
\rho=\left(\begin{array}{cccc}
w_{1} & 0 & 0 & 0 \\
0 & \gamma \gamma^{*} w_{2} & \gamma \delta^{*} w_{2} & 0 \\
0 & \gamma^{*} \delta w_{2} & \delta \delta^{*} w_{2} & 0 \\
0 & 0 & 0 & 0
\end{array}\right)
$$


С помошью техники, развитой в работах [11], можно показать, что в случае выполнения условий

$$
\gamma \delta^{*}-\gamma^{*} \delta=0, \quad\left(\gamma \delta^{*}+\gamma^{*} \delta\right) w_{2}=\left(w_{1}-w_{2}\right)
$$

для состояния (7) выполняется обобщенное неравенство БКХШХ

$$
|E(\vec{a}, \vec{b})+E(\vec{a}, \vec{c})+E(\vec{d}, \vec{b})-E(\vec{d}, \vec{c})| \leqslant 2 \sqrt{2}\left|w_{1}-w_{2}\right| .
$$

Величина "пьюрити" для состояния (7) равна $\mu=w_{1}^{2}+w_{2}^{2}$. С ее помощью неравенство (8) можно записать в виде

$$
|E(\vec{a}, \vec{b})+E(\vec{a}, \vec{c})+E(\vec{d}, \vec{b})-E(\vec{d}, \vec{c})| \leqslant 2 \sqrt{2} \sqrt{2 \mu-1} .
$$

Для чистых состояний $\mu=1$. Мы видим, что, как и в случае соотношений неопределенностей, неравенство БКХШХ (10) для смешанных состояний получается из неравенства БКХШХ (4) для чистых состояний путем умножения на функцию, зависящую от параметра $\mu$.

Мы видим, что при переходе от чистых состояний к смешанным наблюдается ряд закономерностей, общих для соотношений неопределенностей и неравенств БКХШХ. В этой связи представляется целесообразным подробно исследовать вопрос о том, как модифицируется неравенство БКХШХ при переходе от чистых состояний к смешанным. Эта задача дополнительно стимулирована тем, что имеется ряд запутанных состояний, для которых выполняется исходное неравенство Белла. Одним из них является состояние, предложенное Вернером [7]. Это смешанное состояние, и точный квантово-механический расчет показывает, что оно удовлетворяет более сильному неравенству, чем неравенство Белла, полученное в предположении существования в теории СП. Вернеровские состояния сейчас привлекают к себе большое внимание, проблема их генерации обсуждается в работе [12].

В данной работе построены неравенства БКХШХ, явно учитываюшие структуру смешанного состояния. Причем это сделано как в рамках традиционной $\mathrm{KM}$, так и в рамках модели СП. Показано, что в тех случаях, когда смешанное состояние состоит из двух чистых, неравенство БКХШХ для такого состояния можно получить из соответствующего неравенства для чистого состояния, умножая его правую часть на функцию, зависящую от величины $\mu$. Вид этой функции зависит от структуры конкретного состояния. Если же смешанное состояние состоит более чем из двух чистых, то неравенство БКХШХ для такого состояния также можно получить из неравенства для чистого состояния, умножая его правую часть на некоторую функцию. Однако в этом случае данная функция зависит уже не от одного параметра $\mu$, а от всех вероятностей $w_{i}$, с которыми чистые состояния входят в смешанное.

Важным обстоятельством является то, что теория СП исходит из того, что ее предсказания для величины средних значений наблюдаемых должны совпадать с предсказаниями обычной КМ. Это, в частности, справедливо и для среднего значения оператора спиновой корреляции. Поэтому различие между предсказаниями этих теорий возникает только при оценке максимальных значений некоторых линейных комбинаций таких средних. Это обусловлено тем, что при вычислении используется информация о внутренней структуре средних, а она различна в различных теориях. 
В работе [13] было найдено СП-неравенство БКХШХ для смешанных двучастичных спиновых состояний. Его правая часть выражается через вероятности $\mu_{1}, \mu_{2}, \mu_{3}, \mu_{4}$, с которыми чистые состояния входят в смешанное. Это неравенство было получено для случая, когда векторы $\vec{a}, \vec{b}, \vec{c}, \vec{d}$, задающие направления, на которые проецируются спины частиц, образуюших коррелированную пару, лежат в одной плоскости. В данной работе мы обобшим этот результат на случай, когда эти векторы могут иметь произвольные направления в пространстве. Аналогичные неравенства построены и в рамках обычной КМ.

Проведено сравнение полученных неравенств. Показано, что для смешанных состояний разница в предсказаниях, полученных в рамках КМ и модели СП, меньше, чем для чистых состояний. В некоторых специальных случаях предсказания КМ и модели СП совпадают.

\section{3. НЕРАВЕНСТВА БКХШХ В БАЗИСЕ ЗАПУТАННЫХ СОСТОЯНИЙ}

В работах [11] нами были построены обобшенные КМ-неравенства БКХШХ. Их правые части зависят от конкретного состояния, для которого они вычисляются. Оказалось, что сушествуют смешанные запутанные состояния, у которых они сколь угодно малы.

Определим базис в пространстве двучастичных спиновых состояний, который удобно использовать для построения обобщенных СП-неравенств. Обозначим через $|+\rangle$ и $|-\rangle$ векторы одночастичных состояний с проекциями спина на ось $Z$ соответственно $+1 / 2$ и $-1 / 2$. На этих векторах спиновые операторы задаются соотношениями

$$
\begin{aligned}
S_{x}|+\rangle & =\frac{1}{2}|-\rangle, \quad S_{x}|-\rangle=\frac{1}{2}|+\rangle, \quad S_{y}|+\rangle=\frac{i}{2}|-\rangle, \quad S_{y}|-\rangle=-\frac{i}{2}|+\rangle, \\
S_{z}|+\rangle & =\frac{1}{2}|+\rangle, \quad S_{z}|-\rangle=-\frac{1}{2}|-\rangle .
\end{aligned}
$$

В пространстве двучастичных спиновых состояний мы будем использовать базис

$$
\begin{aligned}
\left|\Phi^{1}\right\rangle & =\frac{1}{\sqrt{2}}(|(1)+\rangle|(2)-\rangle-|(1)-\rangle|(2)+\rangle), \\
\left|\Phi^{2}\right\rangle & =\frac{1}{\sqrt{2}}(|(1)+\rangle|(2)-\rangle+|(1)-\rangle|(2)+\rangle), \\
\left|\Phi^{3}\right\rangle & =\frac{1}{\sqrt{2}}(|(1)+\rangle|(2)+\rangle-|(1)-\rangle|(2)-\rangle), \\
\left|\Phi^{4}\right\rangle & =\frac{1}{\sqrt{2}}(|(1)+\rangle|(2)+\rangle+|(1)-\rangle|(2)-\rangle) .
\end{aligned}
$$

При этом операторы $\vec{S}^{(1)}$ действуют на векторы $|(1)+\rangle,|(1)-\rangle$, а операторы $\vec{S}^{(2)}$ - на векторы $|(2)+\rangle,|(2)-\rangle$. Обозначения "(1)" и "(2)" относятся к первой и второй частицам, соответственно.

Неравенства Белла [2] строятся для наблюдаемой величины, называемой спиновой корреляцией. Она определяется как учетверенное среднее значение произведения проекций спина первой частицы на ось $\vec{a}$, задаваемую вектором $\vec{a}$, а второй частишы - на ось $\vec{b}$, задаваемую вектором $\vec{b}$. 
В теории СП [14] предполагается, что каждое состояние описывается совокупностью параметров $\lambda$, принимаюших значения в некотором пространстве $\Lambda$ и характеризующихся функцией распределения $\rho(\lambda)$ такой, что

$$
\int_{\lambda \in \Lambda} \rho(\lambda) d \lambda=1 .
$$

Удвоенное значение проекции спина частицы на ось $\vec{a}$ описывается функцией $\hat{\sigma}_{a}(\lambda)$, которая принимает значения

$$
\hat{\sigma}_{a}(\lambda)= \pm 1 .
$$

В рамках теории СП значение спиновой корреляции $E^{\prime}(\vec{a}, \vec{b})$ описывается выражением

$$
E^{\prime}(\vec{a}, \vec{b})=\int_{\lambda \in \Lambda} d \lambda \rho(\lambda) \hat{\sigma}_{a}(\lambda) \hat{\sigma}_{b}(\lambda) .
$$

Рассмотрим квантово-механическое смешанное состояние, в которое чистые состояния (12) входят с вероятностями $\mu_{i} \geqslant 0, \sum_{i=1}^{4} \mu_{i}=1$. Его матрица плотности имеет вид

$$
\hat{\rho}=\sum_{i=1}^{4} \mu_{i}\left|\Phi^{i}\right\rangle\left\langle\Phi^{i}\right| .
$$

В теории СП при описании смешанных состояний пространство $\Lambda$ разбивают на несколько непересекаюшихся подпространств $\Lambda_{i}$ по числу чистых состояний, входяших в смешанное, таких, что

$$
\int_{\lambda \in \Lambda_{i}} \rho(\lambda) d \lambda=\mu_{i}, \quad \bigcup_{i} \Lambda_{i}=\Lambda, \quad \Lambda_{i} \cap \Lambda_{j}=\varnothing \quad(i \neq j) .
$$

Если при вычислении среднего значения спиновой корреляции для смешанного состояния (16) мы захотим выделить вклад $E^{\prime}(\vec{a}, \vec{b})_{i}$ отдельного чистого состояния $\left|\Phi^{i}\right\rangle$, то нужно ограничить область интегрирования в формуле (15) соответствуюшим подпространством $\Lambda_{i}$ :

$$
E^{\prime}(\vec{a}, \vec{b})_{i}=\int_{\lambda \in \Lambda_{i}} d \lambda \rho(\lambda) \hat{\sigma}_{a}(\lambda) \hat{\sigma}_{b}(\lambda) .
$$

В КМ оператор $\hat{a}$ удвоенной проекции спина на направление, задаваемое вектором $\vec{a}=\left(a_{x}, a_{y}, a_{z}\right)$, имеет вид

$$
\hat{a}=2(\vec{a}, \vec{S})=2\left(a_{x} S_{x}+a_{y} S_{y}+a_{z} S_{z}\right) .
$$

Оператор спиновой корреляции определяется как

$$
\hat{a} \otimes \hat{b}=4\left(\vec{a}, \vec{S}^{(1)}\right) \otimes\left(\vec{b}, \vec{S}^{(2)}\right),
$$

а его среднее значение на состоянии с матрицей плотности $\hat{\rho}=\left\|\rho_{i j}\right\|$ можно представить в виде

$$
E(\vec{a}, \vec{b})=\operatorname{Sp}(\hat{a} \otimes \hat{b} \rho)=\left(a_{x} a_{y} a_{z}\right) P\left(\begin{array}{c}
b_{x} \\
b_{y} \\
b_{z}
\end{array}\right)=(\vec{a}, P \vec{b}),
$$


где $P-(3 \times 3)$-матрица:

$$
\begin{aligned}
P & =\left\|P_{i j}\right\|= \\
& =\left(\begin{array}{ccc}
\rho_{14}+\rho_{23}+\rho_{32}+\rho_{41} & i\left(\rho_{14}-\rho_{23}+\rho_{32}-\rho_{41}\right) & \rho_{13}+\rho_{31}-\rho_{24}-\rho_{42} \\
i\left(\rho_{14}+\rho_{23}-\rho_{32}-\rho_{41}\right) & -\rho_{14}+\rho_{23}+\rho_{32}-\rho_{41} & i\left(\rho_{13}-\rho_{31}-\rho_{24}+\rho_{42}\right) \\
\rho_{12}+\rho_{21}-\rho_{34}-\rho_{43} & i\left(\rho_{12}-\rho_{21}-\rho_{34}+\rho_{43}\right) & \rho_{11}-\rho_{22}-\rho_{33}+\rho_{44}
\end{array}\right)
\end{aligned}
$$

Если смешанное состояние описывается матрицей плотности (16), то можно выделить вклад $E(\vec{a}, \vec{b})_{i}$ отдельного чистого состояния в среднее $(21)$ :

$$
E(\vec{a}, \vec{b})_{i}=\mu_{i} \operatorname{Sp}\left(\hat{a} \otimes \hat{b}\left|\Phi^{i}\right\rangle\left\langle\Phi^{i}\right|\right)=\mu_{i}\left(a_{x} a_{y} a_{z}\right) P_{i}\left(\begin{array}{c}
b_{x} \\
b_{y} \\
b_{z}
\end{array}\right)=\mu_{i}\left(\vec{a}, P_{i} \vec{b}\right) .
$$

С помощью выражения (22) легко найти матрицы $P$ для чистых состояний (12):

$$
\begin{aligned}
P_{1}=\left(\begin{array}{ccc}
-1 & 0 & 0 \\
0 & -1 & 0 \\
0 & 0 & -1
\end{array}\right), & P_{2}=\left(\begin{array}{ccc}
1 & 0 & 0 \\
0 & 1 & 0 \\
0 & 0 & -1
\end{array}\right), \\
P_{3}=\left(\begin{array}{ccc}
-1 & 0 & 0 \\
0 & 1 & 0 \\
0 & 0 & 1
\end{array}\right), & P_{4}=\left(\begin{array}{ccc}
1 & 0 & 0 \\
0 & -1 & 0 \\
0 & 0 & 1
\end{array}\right),
\end{aligned}
$$

Теория СП исходит из того, что ее предсказания для величины средних значений наблюдаемых должны совпадать с предсказаниями обычной КМ [14]. Отсюда следует, что должно выполняться равенство

$$
E^{\prime}(\vec{a}, \vec{b})_{i}=E(\vec{a}, \vec{b})_{i}
$$

или

$$
\int_{\lambda \in \Lambda_{i}} d \lambda \rho(\lambda) \hat{\sigma}_{a}(\lambda) \hat{\sigma}_{b}(\lambda)=\mu_{i} \operatorname{Sp}\left(\hat{a} \otimes \hat{b}\left|\Phi^{i}\right\rangle\left\langle\Phi^{i}\right|\right)=\mu_{i}\left(\vec{a}, P_{i} \vec{b}\right) .
$$

Левая часть неравенства БКХШХ имеет вид

$$
\left|E^{\prime}(\vec{a}, \vec{b})+E^{\prime}(\vec{a}, \vec{c})+E^{\prime}(\vec{d}, \vec{b})-E^{\prime}(\vec{d}, \vec{c})\right| .
$$

Подставляя в выражение (26) соотношения (18), (25) и учитывая (14), получаем

$$
\begin{gathered}
\left|\int_{\lambda \in \Lambda_{i}} d \lambda \rho(\lambda)\left[\hat{\sigma}_{a}(\lambda)\left[\hat{\sigma}_{b}(\lambda)+\hat{\sigma}_{c}(\lambda)\right]+\hat{\sigma}_{d}(\lambda)\left[\hat{\sigma}_{b}(\lambda)-\hat{\sigma}_{c}(\lambda)\right]\right]\right|= \\
=\left|E^{\prime}(\vec{a}, \vec{b})_{i}+E^{\prime}(\vec{a}, \vec{c})_{i}+E^{\prime}(\vec{d}, \vec{b})_{i}-E^{\prime}(\vec{d}, \vec{c})_{i}\right| \leqslant 2 \mu_{i} .
\end{gathered}
$$

Если в выражении (27) распространить процедуру интегрирования на все пространство СП $\Lambda$, то в левой части этого неравенства возникнет сумма

$$
\sum_{i=1}^{4}\left|E^{\prime}(\vec{a}, \vec{b})_{i}+E^{\prime}(\vec{a}, \vec{c})_{i}+E^{\prime}(\vec{d}, \vec{b})_{i}-E^{\prime}(\vec{d}, \vec{c})_{i}\right|
$$


Мы предположили, что для каждой пары частиц выполняется равенство (25), т.е. СПзначения спиновой корреляции равны КМ-значениям спиновой корреляции. В работе [13] рассматривался случай, когда все четыре вектора $\vec{a}, \vec{b}, \vec{c}, \vec{d}$ лежат в плоскости $(x z)$. Для векторов $\vec{v}$, лежащих в этой плоскости, выполняется соотношение

$$
\left(P_{1}+P_{4}\right) \vec{v}=\left(P_{2}+P_{3}\right) \vec{v}=0 .
$$

Это следует непосредственно из вида $(24)$ матриц $P_{i}$.

Из соотношений (29) и формулы (25) получаем

$$
\begin{aligned}
& \operatorname{Sp}\left(\hat{a} \otimes \hat{b}\left|\Phi^{1}\right\rangle\left\langle\Phi^{1}\right|\right)+\operatorname{Sp}\left(\hat{a} \otimes \hat{b}\left|\Phi^{4}\right\rangle\left\langle\Phi^{4}\right|\right)=0, \\
& \operatorname{Sp}\left(\hat{a} \otimes \hat{b}\left|\Phi^{2}\right\rangle\left\langle\Phi^{2}\right|\right)+\operatorname{Sp}\left(\hat{a} \otimes \hat{b}\left|\Phi^{3}\right\rangle\left\langle\Phi^{3}\right|\right)=0 .
\end{aligned}
$$

Из формул (18), (25) и (30) следует, что

$$
E^{\prime}(\vec{a}, \vec{b})=\frac{\mu_{1}-\mu_{4}}{\mu_{1}} \int_{\lambda \in \Lambda_{1}} d \lambda \rho(\lambda) \hat{\sigma}_{a}(\lambda) \hat{\sigma}_{b}(\lambda)+\frac{\mu_{2}-\mu_{3}}{\mu_{2}} \int_{\lambda \in \Lambda_{2}} d \lambda \rho(\lambda) \hat{\sigma}_{a}(\lambda) \hat{\sigma}_{b}(\lambda) .
$$

Подставляя выражение (31) в формулу (27), получим обобщенное СП-неравенство БКХШХ для смешанного состояния (16):

$$
\left|E^{\prime}(\vec{a}, \vec{b})+E^{\prime}(\vec{a}, \vec{c})+E^{\prime}(\vec{d}, \vec{b})-E^{\prime}(\vec{d}, \vec{c})\right| \leqslant 2\left(\left|\mu_{1}-\mu_{4}\right|+\left|\mu_{2}-\mu_{3}\right|\right) .
$$

Неравенство (32) было получено в работе [13]. Его недостатком является то, что оно выведено в предположении того, что векторы $\vec{a}, \vec{b}, \vec{c}, \vec{d}$ лежат в плоскости $(x z)$. Этот результат можно обобшить, используя технику редушированной матришы плотности [11].

Из вида матрищ (24) следует, что для векторов $\vec{a}$, лежащих в плоскости $(x y)$, вьполняется соотношение

$$
\left(P_{1}+P_{2}\right) \vec{a}=\left(P_{3}+P_{4}\right) \vec{a}=0,
$$

а для векторов $\vec{b}$, лежаших в плоскости $(y z),-$ соотношение

$$
\left(P_{1}+P_{3}\right) \vec{b}=\left(P_{2}+P_{4}\right) \vec{b}=0 .
$$

Из соотношения (33) следует, что если векторы $\vec{a}, \vec{b}, \vec{c}, \vec{d}$ лежат в плоскости $(x y)$, то для смешанного состояния (16) выполняется СП-неравенство БКХШХ

$$
\left|E^{\prime}(\vec{a}, \vec{b})+E^{\prime}(\vec{a}, \vec{c})+E^{\prime}(\vec{d}, \vec{b})-E^{\prime}(\vec{d}, \vec{c})\right| \leqslant 2\left(\left|\mu_{1}-\mu_{2}\right|+\left|\mu_{3}-\mu_{4}\right|\right) .
$$

Если же векторы $\vec{a}, \vec{b}, \vec{c}, \vec{d}$ лежат в плоскости (yz), то СП-неравенство БКХшХ для смешанного состояния (16) принимает вид

$$
\left|E^{\prime}(\vec{a}, \vec{b})+E^{\prime}(\vec{a}, \vec{c})+E^{\prime}(\vec{d}, \vec{b})-E^{\prime}(\vec{d}, \vec{c})\right| \leqslant 2\left(\left|\mu_{1}-\mu_{3}\right|+\left|\mu_{2}-\mu_{4}\right|\right) .
$$

Рассмотрим теперь ту же задачу с точки зрения обобщенных КМ-неравенств БКХШХ [6], [7]. Они имеют вид

$$
|E(\vec{a}, \vec{b})+E(\vec{a}, \vec{c})+E(\vec{d}, \vec{b})-E(\vec{d}, \vec{c})| \leqslant \sqrt{2} \sup _{\vec{n}_{1}, \vec{n}_{2}}\left(\left\|P\left(\vec{n}_{1}\right)\right\|+\left\|P\left(\vec{n}_{2}\right)\right\|\right) .
$$


Здесь sup берется по всем парам векторов $\vec{n}_{1}, \vec{n}_{2}$ таким, что

$$
\left(\vec{n}_{1}, \vec{n}_{2}\right)=0, \quad\left\|\vec{n}_{1}\right\|=\left\|\vec{n}_{2}\right\|=1 .
$$

Для состояния (16) матриша $P$ вычисляется с помошью формулы (22):

$$
P=\left(\begin{array}{ccc}
-\mu_{1}+\mu_{2}-\mu_{3}+\mu_{4} & 0 & 0 \\
0 & -\mu_{1}+\mu_{2}+\mu_{3}-\mu_{4} & 0 \\
0 & 0 & -\mu_{1}-\mu_{2}+\mu_{3}+\mu_{4}
\end{array}\right) .
$$

Если векторы $\vec{a}, \vec{b}, \vec{c}, \vec{d}$ лежат в плоскости $(x z)$, то правая часть неравенства (37) достигает своего максимального значения на векторах

$$
\vec{n}_{1}=\frac{1}{\sqrt{2}}\left(\begin{array}{c}
1 \\
0 \\
-1
\end{array}\right), \quad \vec{n}_{2}=\frac{1}{\sqrt{2}}\left(\begin{array}{l}
1 \\
0 \\
1
\end{array}\right) .
$$

Это максимальное значение равно $2 \sqrt{2} \sqrt{\left(\mu_{1}-\mu_{4}\right)^{2}+\left(\mu_{2}-\mu_{3}\right)^{2}}$. С учетом данного выражения получаем, что если векторы $\vec{a}, \vec{b}, \vec{c}, \vec{d}$ лежат в плоскости $(x z)$, то КМ-неравенство БКХШХ (37) принимает вид

$$
|E(\vec{a}, \vec{b})+E(\vec{a}, \vec{c})+E(\vec{d}, \vec{b})-E(\vec{d}, \vec{c})| \leqslant 2 \sqrt{2} \sqrt{\left(\mu_{1}-\mu_{4}\right)^{2}+\left(\mu_{2}-\mu_{3}\right)^{2}} .
$$

Аналогично получаем, что если векторы $\vec{a}, \vec{b}, \vec{c}, \vec{d}$ лежат в плоскости $(x y)$, то неравенство (37) принимает вид

$$
|E(\vec{a}, \vec{b})+E(\vec{a}, \vec{c})+E(\vec{d}, \vec{b})-E(\vec{d}, \vec{c})| \leqslant 2 \sqrt{2} \sqrt{\left(\mu_{1}-\mu_{2}\right)^{2}+\left(\mu_{3}-\mu_{4}\right)^{2}},
$$

а если в плоскости $(y z)$, то вид

$$
|E(\vec{a}, \vec{b})+E(\vec{a}, \vec{c})+E(\vec{d}, \vec{b})-E(\vec{d}, \vec{c})| \leqslant 2 \sqrt{2} \sqrt{\left(\mu_{1}-\mu_{3}\right)^{2}+\left(\mu_{2}-\mu_{4}\right)^{2}} .
$$

Сравним правые части СП-неравенств БКХШХ (32), (35), (36) с правыми частями KM-неравенств (40), (42). Легко видеть, что правые части КМ-неравенств всегда больше или равны правым частям соответствуюших СП-неравенств, причем равенство достигается при

$$
\left(\mu_{1}-\mu_{4}\right)^{2}=\left(\mu_{2}-\mu_{3}\right)^{2}, \quad\left(\mu_{1}-\mu_{2}\right)^{2}=\left(\mu_{3}-\mu_{4}\right)^{2}, \quad\left(\mu_{1}-\mu_{3}\right)^{2}=\left(\mu_{2}-\mu_{4}\right)^{2} .
$$

Для чистых состояний эти условия никогда не выполняются, т.е. теория СП и КМ для смешанных состояний дают более близкие результаты, чем для чистых.

До сих пор мы предполагали, что все четыре вектора $\vec{a}, \vec{b}, \vec{c}, \vec{d}$, используюшиеся при построении неравенств, лежат в одной плоскости. Рассмотрим теперь общую ситуацию, когда на эти векторы не наложено никаких ограничений.

Как и раншше, построим сначала СП-неравенства БКХШХ, соответствуюшие смешанному состоянию (16). Для этого воспользуемся соотношением

$$
P_{1}+P_{2}+P_{3}+P_{4}=0
$$


между матрицами $P_{i}$, отвечающими векторам базиса (12). Оно следует непосредственно из их явного вида (24). С его помошью можно получить неравенство

$$
\left|E^{\prime}(\vec{a}, \vec{b})+E^{\prime}(\vec{a}, \vec{c})+E^{\prime}(\vec{d}, \vec{b})-E^{\prime}(\vec{d}, \vec{c})\right| \leqslant 2 A\left(w_{1}, w_{2}, w_{3}, w_{4}\right),
$$

где

$$
\begin{array}{r}
A\left(w_{1}, w_{2}, w_{3}, w_{4}\right)=\min \left(\left|w_{1}-w_{4}\right|+\left|w_{2}-w_{4}\right|+\left|w_{3}-w_{4}\right|,\right. \\
\left|w_{1}-w_{3}\right|+\left|w_{2}-w_{3}\right|+\left|w_{4}-w_{3}\right|, \\
\left|w_{1}-w_{2}\right|+\left|w_{3}-w_{2}\right|+\left|w_{4}-w_{2}\right|, \\
\left.\left|w_{2}-w_{1}\right|+\left|w_{3}-w_{1}\right|+\left|w_{4}-w_{1}\right|\right) .
\end{array}
$$

Аналогичным образом для КМ-неравенств БКХШХ с помошью соотношения (44) получаем

$$
|E(\vec{a}, \vec{b})+E(\vec{a}, \vec{c})+E(\vec{d}, \vec{b})-E(\vec{d}, \vec{c})| \leqslant 2 \sqrt{2} B\left(w_{1}, w_{2}, w_{3}, w_{4}\right)
$$

где

$$
\begin{aligned}
B\left(w_{1}, w_{2}, w_{3}, w_{4}\right)=\max ( & \sqrt{\left(w_{1}-w_{4}\right)^{2}+\left(w_{2}-w_{3}\right)^{2}}, \\
& \left.\sqrt{\left(w_{1}-w_{2}\right)^{2}+\left(w_{3}-w_{4}\right)^{2}}, \sqrt{\left(w_{1}-w_{3}\right)^{2}+\left(w_{2}-w_{4}\right)^{2}}\right) .
\end{aligned}
$$

Таким образом, в случае смешанных состояний типа (16) экспериментальные данные следует сравнивать со значениями величин $A$ и $B$. В ряде случаев эти значения близки друг к другу, а иногда, как показывают соотношения (43), даже совпадают.

Также следует иметь в виду, что вид правых частей неравенств БКХШХ зависит не только от тех вероятностей, с которыми чистые состояния входят в смешанное, но и от выбора этих чистых состояний. Если выбрать другие чистые состояния, то изменится как само смешанное состояние, так и соответствующие ему неравенства. Проиллюстрируем это на примере.

\section{4. НЕРАВЕНСТВА БКХШХ В БАЗИСЕ ФАКТОРИЗУЕМЫХ СОСТОЯНИЙ}

В теории СП предполагаются известными не только общий спин пары частиц, но и проекции спинов отдельных частиц, входящих в эту пару. В обычной КМ таким свойством обладают факторизуемые состояния. По этой причине для факторизумых состояний СП-неравенства БКХШХ должны совпадать с КМ-неравенствами. Для чистых состояний это утверждение хорошо известно. Покажем теперь, что оно справедливо и для смешанных состояний.

Вместо базиса (12), образованного запутанными состояниями $\left|\Phi^{i}\right\rangle$, используем базис, состояший из факторизуемых состояний $\left|\Psi^{i}\right\rangle$ :

$$
\begin{aligned}
\left|\Psi^{1}\right\rangle & =|(1)+\rangle|(2)+\rangle, & & \left|\Psi^{2}\right\rangle=|(1)+\rangle|(2)-\rangle, \\
\left|\Psi^{3}\right\rangle & =|(1)-\rangle|(2)+\rangle, & & \left|\Psi^{4}\right\rangle=|(1)-\rangle|(2)-\rangle,
\end{aligned}
$$


а вместо смешанного состояния (16) рассмотрим смешанное состояние

$$
\hat{\rho}=\sum_{i=1}^{4} \nu_{i}\left|\Psi^{i}\right\rangle\left\langle\Psi^{i}\right|
$$

Чистые состояния (47) входят в него с вероятностями $\nu_{1}, \nu_{2}, \nu_{3}, \nu_{4}$. Это так называемое разделяемое состояние. Из обших соображений следует, что для таких состояний СП-неравенства БКХШХ должны совпадать с КМ-неравенствами. Докажем это утверждение с помошью прямого вычисления.

Сначала проведем расчет в рамках традиционной КМ. Матрица плотности $\rho$ смешанного состояния (48) и его $P$-матрица имеют вид

$$
\rho=\frac{1}{2}\left(\begin{array}{cccc}
\nu_{1} & 0 & 0 & 0 \\
0 & \nu_{2} & 0 & 0 \\
0 & 0 & \nu_{3} & 0 \\
0 & 0 & 0 & \nu_{4}
\end{array}\right), \quad P=\left(\begin{array}{ccc}
0 & 0 & 0 \\
0 & 0 & 0 \\
0 & 0 & \nu_{1}-\nu_{2}-\nu_{3}+\nu_{4}
\end{array}\right)
$$

Для такой матришы $P$ КМ-неравенство (37) принимает вид

$$
|E(\vec{a}, \vec{b})+E(\vec{a}, \vec{c})+E(\vec{d}, \vec{b})-E(\vec{d}, \vec{c})| \leqslant 2\left|\nu_{1}-\nu_{2}-\nu_{3}+\nu_{4}\right|
$$

Найдем теперь СП-неравенство БКХшХ, отвечаюшее состоянию (48). С этой целью определим сначала $P$-матрищы векторов $\left|\Psi^{i}\right\rangle$ из базиса (47). В соответствии с формулой (22) имеем

$$
\begin{array}{ll}
P_{1}=\left(\begin{array}{ccc}
0 & 0 & 0 \\
0 & 0 & 0 \\
0 & 0 & 1
\end{array}\right), & P_{2}=\left(\begin{array}{ccc}
0 & 0 & 0 \\
0 & 0 & 0 \\
0 & 0 & -1
\end{array}\right), \\
P_{3}=\left(\begin{array}{ccc}
0 & 0 & 0 \\
0 & 0 & 0 \\
0 & 0 & -1
\end{array}\right), & P_{4}=\left(\begin{array}{lll}
0 & 0 & 0 \\
0 & 0 & 0 \\
0 & 0 & 1
\end{array}\right)
\end{array}
$$

Из формул (51) следуют соотношения

$$
P_{1}=P_{4}, \quad P_{2}=P_{3}, \quad P_{1}=-P_{2},
$$

являющиеся аналогом соотношений (29), (33), (34) для базиса (12).

Как и в случае базиса (12), в теории СП предполагается, что каждое базисное состояние $\left|\Psi^{i}\right\rangle$ описывается совокупностью параметров $\lambda$ таких, что

$$
\int_{\lambda \in \Lambda_{i}} \rho(\lambda) d \lambda=\nu_{i}
$$

Для СП-значения спиновой коррелящии $E^{\prime}(\vec{a}, \vec{b})$ с помощью соотношений $(52)$ получим выражение

$$
E^{\prime}(\vec{a}, \vec{b})=\frac{\nu_{1}-\nu_{2}-\nu_{3}+\nu_{4}}{\nu_{1}} \int_{\lambda \in \Lambda_{1}} d \lambda \rho(\lambda) \hat{\sigma}_{a}(\lambda) \hat{\sigma}_{b}(\lambda) .
$$


Отсюда получаем СП-неравенство БКХШХ для состояния (48):

$$
\left|E^{\prime}(\vec{a}, \vec{b})+E^{\prime}(\vec{a}, \vec{c})+E^{\prime}(\vec{d}, \vec{b})-E^{\prime}(\vec{d}, \vec{c})\right| \leqslant 2\left|\nu_{1}-\nu_{2}-\nu_{3}+\nu_{4}\right| .
$$

Мы видим, что правые части выражений (50) и (55) совпадают, что согласуется с обшими требованиями теории СП.

Из формул (41), (42), (45), (46), (55) видно, что при некоторых соотношениях между вероятностями $\mu_{i}, \nu_{i}$ правые части этих неравенств обрашаются в нуль:

$$
|E(\vec{a}, \vec{b})+E(\vec{a}, \vec{c})+E(\vec{d}, \vec{b})-E(\vec{d}, \vec{c})|=0 .
$$

Во всех этих случаях обрашение в нуль правой части КМ-неравенства БКХШХ обусловлено тем, что в нуль обрашаются значения спиновой корреляции $E(\vec{a}, \vec{b})$. Последнее, в свою очередь, является следствием того, что для таких состояний $P$-матрицы (22) тождественно равны нулю. В работе [13] было найдено состояние, удовлетворяющее равенству (56). Однако его матрица плотности пропорциональна единичной. Используя технику $P$-матрицы, можно легко описать все двучастичные состояния, в том числе и с неединичными матрицами плотности, для которых выполняется равенство (56). В работах [11] было показано, что любой матрице $P$ соответствует 6 -параметрическое семейство матриц плотности $\rho$. В случае нулевой матрицы $P$ эти матрицы $\tilde{\rho}_{0}$ имеют вид

$$
\tilde{\rho}_{0}=\frac{1}{4}\left(\begin{array}{cccc}
1+A_{1}+A_{2} & A_{12}+i B_{12} & A_{13}+i B_{13} & 0 \\
A_{12}-i B_{12} & 1-A_{1}+A_{2} & 0 & A_{13}+i B_{13} \\
A_{13}-i B_{13} & 0 & 1+A_{1}-A_{2} & A_{12}+i B_{12} \\
0 & A_{13}-i B_{13} & A_{12}-i B_{12} & 1-A_{1}-A_{2}
\end{array}\right) .
$$

Здесь $A_{1}, A_{2}, A_{12}, B_{12}, A_{13}, B_{13}$ - произвольные вешественные параметры, область изменения которых ограничивается тем условием, что матрица (57) должна иметь физический смысл. Среди состояний (57) факторизуемыми являются те, для которых выполняется дополнительное условие $A_{2}=A_{13}=B_{13}=0$. Чистых состояний среди них нет, все они смешанные. Отметим, что из вида обобщенного неравенства БКХШХ (37) сразу следует, что при ненулевой матрице $P$ его правая часть никогда не обратится в нуль.

\section{5. ЗАКЛЮЧЕНИЕ}

Мы показали, что вид неравества БКХШХ определяется структурой состояния, которое используется в эксперименте, в частности зависит от того, является оно чистым или смешаным, и если смешаным, то из каких чистых оно состоит. Причем в некоторых случаях разница между неравенствами, полученными в рамках обычной КМ и теории СП, может быть невелика. Кроме того, результат измерения величины спиновой корреляции сушественным образом зависит от того, как расположены в пространстве векторы $\vec{a}, \vec{b}, \vec{c}, \vec{d}$. Так, например, в случае состояния (48) из вида его $P$-матрицы (49) сразу получаем, что если данные векторы лежат в плоскости $(x y)$, то эта величина равна нулю. Отсюда следует, что, зная структуру состояния, можно так выбрать векторы $\vec{a}, \vec{b}$, $\vec{c}, \vec{d}$, чтобы разница предсказаний КМ и теории СП была наибольшей. Поэтому проверку неравенств БКХШХ нужно начинать с исследования структуры того состояния, 
которое используется в эксперименте. Это можно делать с помошью методов квантовой томографии [15]. Определив структуру состояния, т.е. установив, какие чистые состояния и с какими вероятност ями входят в него, надо найти явньй вид СП- и КМ-неравенств БКХШХ. Данная процедура сводится к вычислению правых частей соответствующих неравенств, при этом используются соотношения между $P$-матришами чистых состояний. И только после этого, когда уже известен явный вид неравенств, которые мы собираемся проверять, и те направления векторов, которые обеспечивают наибольшую разницу предсказаний КМ и теории СП, следует переходить непосредственно к процедуре проверки.

\section{Список литературы}

[1] W. Heisenberg. Z. Phys. 1927. V. 43. P. 172.

[2] J. S. Bell. Physics. 1964. V. 1. P. 195.

[3] А. Ю. Хренников. Неколмогоровские теории вероятностей и квантовая физика. М.: Физматлит, 2003; A. S. Holevo. Statistical Structure of Quantum Theory. Berlin-Heidelberg: Springer, 2001; L. Accardi, Yu. G. Lu, I. V. Volovich. Quantum Theory and Its Stochastic Limit. Berlin-Heidelberg: Springer, 2002; I. Volovich, Ya. Volovich. Bell's theorem and random variables. quant-ph/0009058; A. Khrennikov, I. Volovich. Einstein, Podolsky and Rosen versus Bohm and Bell. quant-ph/0211078; A. Khrennikov. Found. Phys. 2002. V. 32. P. 1159.

[4] A. Khrennikov. Contextual approach to quantum mechanics and the theory of the fundamental prespace. V. 1. quant-ph/0306003.

[5] J. F. Clauser, M. A. Horne, A. Shimony, R. A. Holt. Phys. Rev. Lett. 1969. V. 23. P. 880.

[6] H. F. Hofmann, S. Takeuchi. Phys. Rev. A. 2003. V. 68. P. 032103; quant-ph/0212090.

[7] R. F. Werner. Phys. Rev. A. 1989. V. 40. P. 4277.

[8] E. H. Kennard. Z. Phys. 1927. V. 44. P. 326.

[9] Г. Вейль. Теория групп и квантовая механика. М.: Наука, 1986.

[10] В. В. Додонов, В. И. Манько. Тр. ФИАН. 1987. Т. 183. С. 3.

[11] B. А. Андреев, В. И. Манько. Письма в ЖЭТФ. 2000. Т. 72. С. 130; V. A. Andreev, V. I. Man'ko. Phys. Lett. A. 2000. V. 281. P. 278.

[12] M. Barbieri, F. De Martini, G. Di Nepi, P. Mataloni. Violation of Bell inequalities and quantum tomography with pure-states, Werner-states and maximally entangled mixed states created by a universal quantum entangler. quant-ph/0303018.

[13]X.-H. Wu, H.-S. Zong, H.-R. Pong, F. Wang. Phys. Rev. A. 2001. V. 64. P. 022103(3).

[14]F. J. Belinfante. A Survey of Hidden-Variables Theories. Oxford-New York: Pergamon, 1973.

[15] В. А. Андреев, В. И. Манько. ЖЭТФ. 1998. Т. 114. С. 437; V. A. Andreev, V. I. Man'ko. J. Opt. B: Quantum Semiclass. Opt. 2000. V. 2. P. 122.

Поступила в редакцию 3.IX.2003 г. 